\title{
Multislice Computed Tomography Imaging of Total Anomalous Pulmonary Venous Circulation: Three Rare Case Reports
}

\author{
Nusrat Ghafoor ${ }^{1}$, Md. Rokonujjaman Selim ${ }^{2}$, Nawshin Siraj ${ }^{3}$
}

\begin{abstract}
:
Total anomalous pulmonary venous connection is a rare congenital cardiac anomaly in which the pulmonary veins have no connection with the left atrium and connect directly to the right atrium or to one of the systemic veins. In the diagnosis of total anomalous pulmonary venous connection, the presence and severity of the venous obstruction is important. Computed Tomography (CT) imaging is known to be a useful method for the evaluation of total anomalous pulmonary venous connection. Here we report 3 cases of total anomalous pulmonary venous circulation (TAPVC) where 32 slice CT scan played an important role in pre-operative diagnosis and management. Age of the patients ranged from seven months to sixteen years. Symptoms of the patients' varied from fatigue, shortness of breath, mild cyanosis and reduced growth for their age. Echocardiography done revealed dilated right cardiac chambers and atrial septal defect (ASD) with suspicion of TAPVC. CT scan was performed and it provided detailed information about intra-cardiac anatomy, pulmonary veins and their confluence, route, drainage and most importantly their distance and relation with the left atrium. It also provided information about coronary sinus and its drainage. This information is very important for surgical mapping and planning.
\end{abstract}

Key words: Multi-slice, CT, Pulmonary, Venous, Anomalies

\section{Introduction:}

Total anomalous pulmonary venous connection (TAPVC) is a complex congenital cardiac anomaly in which all 4 pulmonary veins drain into systemic veins or the right atrium with or without pulmonary venous obstruction. Systemic and pulmonary venous blood mix in the right atrium. An atrial defect or foramen ovale (part of the complex) is important in left ventricular output both in fetal and in newborn circulation. ${ }^{1}$ Total anomalous pulmonary venous connection (TAPVC) is a relatively uncommon congenital defect representing approximately $2 \%$ of all congenital heart anomalies. ${ }^{2}$

Echocardiography (ECHO) is the mainstay of diagnosis of congenital heart disease. Its strength includes an absence of radiation, the ability to evaluate intra-cardiac structure and function and the ability to perform hemodynamic assessment. However, it is limited in the evaluation of certain portions of the aorta (particularly the ascending aorta and the transverse arch), the distal pulmonary arteries, the right ventricle, and the pulmonary veins. ${ }^{3}$ The traditional method of diagnosis of congenital heart disease has been the catheter angiography. While effective in diagnosis, angiography is an invasive procedure. Evaluation of right and left-sided defects may require both venous and arterial punctures. ${ }^{4}$

The development of multi-slice computed tomography (MSCT) has overcome these barriers thus increasing its use in cardiac imaging in patients with pulmonary venous anomalies. Multi-slice CT has the advantages of fast scan speed, high spatial resolution, enabling acquisition of isotropic volume data, and simultaneous

\section{Authors' information:}

${ }^{1}$ Dr. Nusrat Ghafoor, Associate Consultant, Department of Radiology \& Imaging, Ibrahim Cardiac Hospital \& Research Institute, Dhaka

${ }^{2}$ Md. Rokonujjaman Selim, Associate Professor \& consultant, Department of Cardiovascular \& thoracic surgery, Ibrahim Cardiac Hospital \& Research Institute, Dhaka

${ }^{3}$ Dr. Nawshin Siraj, Senior Consultant \& Head of the Department of Radiology \& Imaging, Ibrahim Cardiac Hospital \& Research Institute, Dhaka Address of correspondence: Dr. Nusrat Ghafoor, Cell Phone: +880 1711316595 Email: ghafoornusrat@yahoo.com 
evaluation of airways and lung parenchyma, thus increasing the ability to answer most clinical questions about structural abnormalities in patients with congenital heart disease. ${ }^{5}$ Our aim was to assess the role of MSCT angiography in the evaluation of extra-cardiac vascular abnormalities in pulmonary venous anomalies.

\section{Patients \& Methods:}

This case series study included 3 patients suspected of having pulmonary venous anomalies on examination and echocardiography. Their age ranged from 7 months to 16 years. All patients were initially examined by echocardiography and were proved or suspected by echocardiography to have pulmonary venous anomalies. After that they were referred to Ibrahim Cardiac Hospital \& Research Institute, Dhaka for CT angiography of the heart and thoracic vessels to delineate detail cardiac anatomy, extra cardiac vessels and pulmonary abnormality. Consultation with the referring physician was attempted prior to the study to know the clinical background of the case and delineate specific questions raised by the physician in order to understand the reason for referral and expectations of the physician from the referral centre. Review of echocardiographic reports was done if available. Renal function was checked and history was taken from the parents.

\section{Technique of examination}

Scanning and post-procedural observation: All studies were performed using a General Electronics (GE) Light Speed 32 Slice Machine. The patient was kept in sedation by minor general anesthesia. A scanogram was obtained from the patients while they were lying on the CT table in supine position from the root of the neck including the proximal aspects of the common carotid and subclavian arteries down to the level of the portal vein inferiorly. This range is important to detect associated aortic arch branch anomalies, situs inversus, situs ambiguous, abdominal aortic coarctation and infra-diaphragmatic type of total anomalous pulmonary venous drainage. Electrocardiography gating was not performed to avoid unnecessary excess radiation exposure to the patient. Non-diluted, Non-ionic contrast material was used (Iopamidol 370) at the rate of 2-3 $\mathrm{ml} / \mathrm{kg}$. The low dose protocol (80 - $120 \mathrm{kVp}$ ) was followed with $\mathrm{mA}$ being adjusted according to the patient's body-weight. The slice thickness and pitch were 1.2 and $0.96 \mathrm{~mm}$ respectively. The patient was kept under observation for 15-30 min after the procedure till recovery from sedation. Nothing was given by mouth till complete recovery. The peripheral venous line was then removed.

Image reconstruction, post processing and interpretation: All images were transferred to the workstation for post-processing. Maximum intensity projections (MIP), three dimensional volume rendering (VR), multiplanar (MPR) and curved planer reformations (CPR) were created at different angles of views. Images, which included cardiac findings and extra-cardiac findings (lung fields, pleural sacs and upper abdominal cut-abnormalities) were interpreted guided by the anatomical and segmental / sequential approach.

\section{Case Reports:}

\section{Case: 1}

A 16-year old male patient presented with exertional chest pain of 5 years duration. He was mildly cyanotic with oxygen saturation $\mathrm{SpO}_{2} 90 \%$. His all biochemical records and chest X-ray were within normal limit. ECG showed right ventricular hypertrophy $(\mathrm{RVH})$. ECHO revealed TAPVC and moderate atrial septal defect (ASD). He also had tricuspid regurgitation (TR), pulmonary hypertension $(\mathrm{PH})$ and dilated Right atrium-Right ventricle. But ECHO could not provide detail anatomy which was needed for surgery. Then cardiac surgeon asked for CT cardiac angiogram which showed all dilated pulmonary veins forming a confluence just behind the small left atrium (LA) and then goes upwards as vertical vein and joins with brachiocephalic vein and then drains into dilated SVC. There was Large ASD, dilated right heart and small LA. Lung vascularity was increased. Other cardiac structures were normal. CT diagnosis was supracardiac TAPVC. Operative finding is same. Now the patient is doing well. 
Figure $1.1 \&$ 1.2: Coronal oblique 3D volumerendered (VRT) \& Maximum Intensity Projection (MIP) image shows the confluence of the pulmonary veins into a common channel, which in turn, drains into the SVC- Supracardiac variety TAPVC

\section{Case-1}

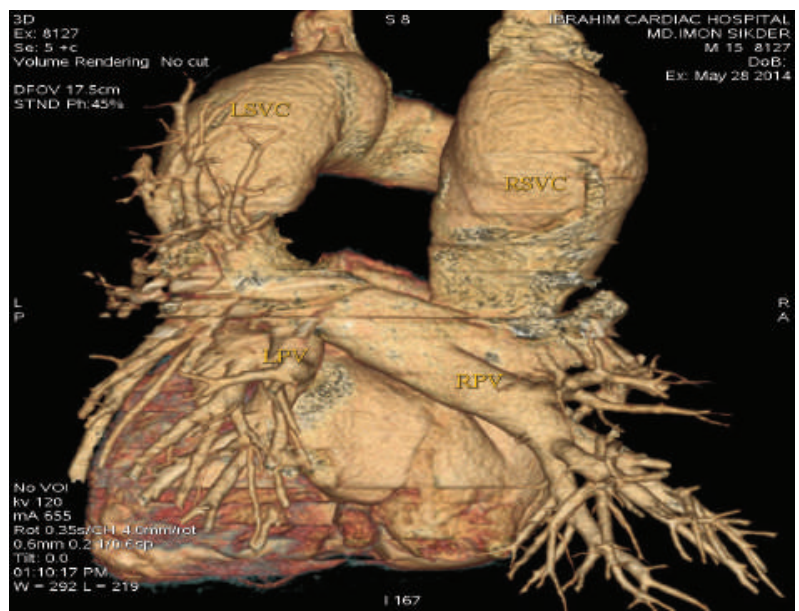

Figure : 1.1

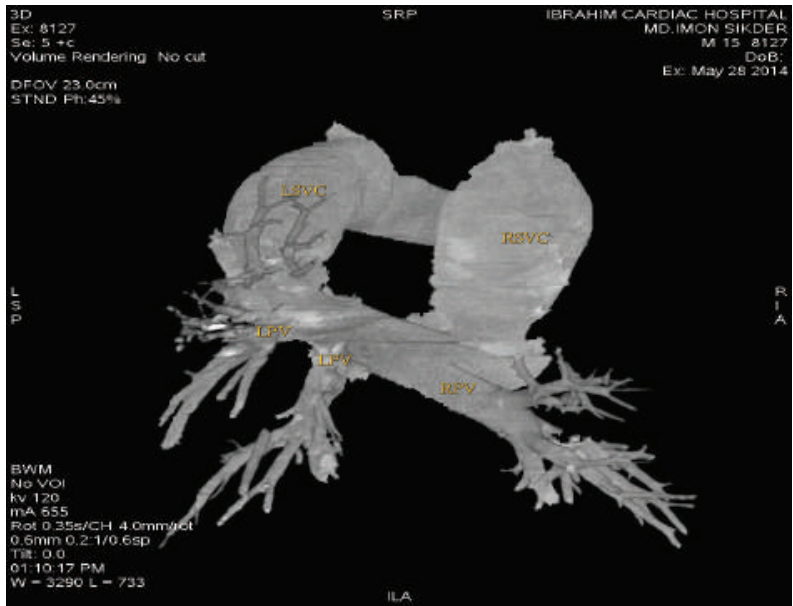

Figure : 1.2

\section{Case: 2}

This 7-month old male patient had shortness of breath and bluish skin while crying. He was mildly cyanotic \& tachypnic with oxygen saturation (SpO2) being $80 \%$. His all biochemical records and ECG were normal. But cardiomegaly was noted on chest $\mathrm{X}$-ray. ECHO findings revealed large ASD with suspicion of TAPVC. The preoperative CT cardiac angiogram done showed all pulmonary veins to form a confluence draining into the RA through dilated coronary sinus with large ASD and dilated right heart chamber. Lung vascularity was increased. CT diagnosis was supracardiac TAPVC. Other cardiac structures were normal. Operative findings put concurrence with the CT report. The patient is doing well after operation.

\section{Case-2}

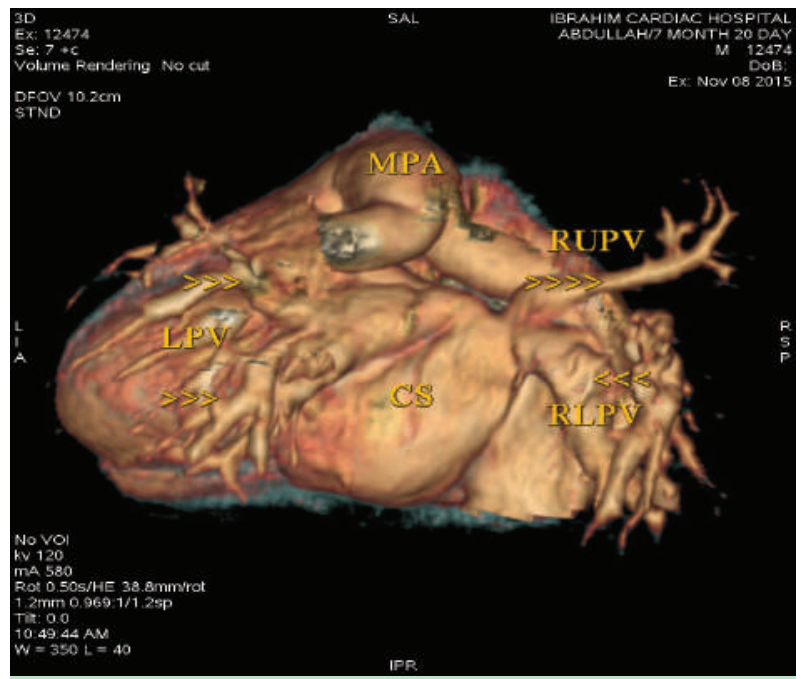

Figure : 2.1

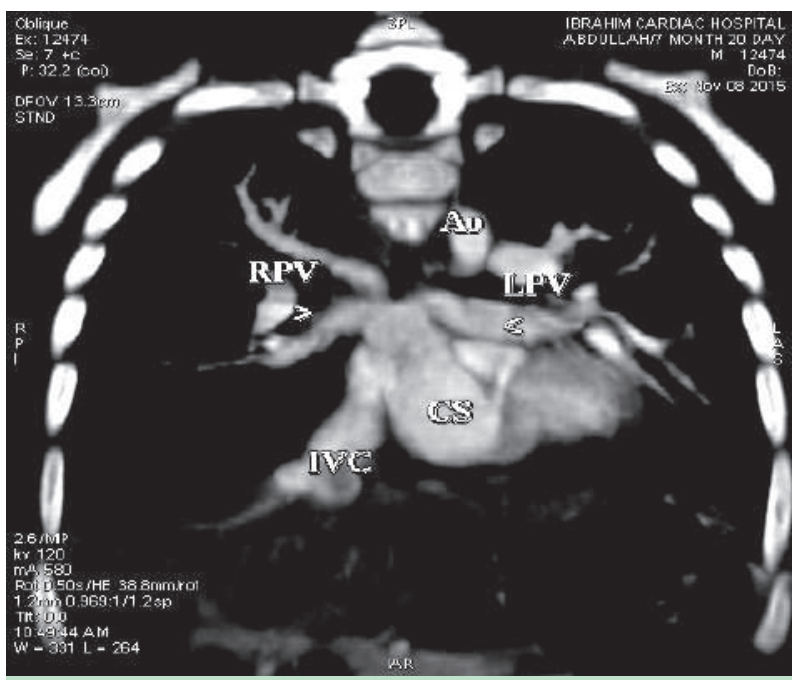

Figure : 2.2

Figure 2.1 \& 2.2: 3D volume-rendered (VRT) image and Multiplanar reconstruction (MPR) 
Coronal oblique image show the confluence of the pulmonary veins into a common channel, which in turn, drains into the dilated Coronary sinus Intracardiac variety TAPVC

\section{Case: 3}

A 9-month old infant presented with shortness of breath, feeding problems, repeated pneumonia sick appearance and cyanosis. He was underweight. He had mildly reduced breath sound and was mildly cyanotic \& tachypnic with $\mathrm{SpO} 2$ $80 \%$. His all biochemical records were within normal range. ECG was indicative of RVH and chest X-ray exhibited cardiomegaly. ECHO shows TAPVC, large Persistent Foramina Ovale (PFO), Mild Tricuspid Regurgitation (TR) \& Moderate Pulmonary Hypertension ( $\mathrm{PH})$. The preoperative CT cardiac angiogram demonstrated two right pulmonary veins to form a confluence and drains into RA through dilated coronary sinus. Left upper pulmonary vein drains into superior venacava (SVC) as vertical vein. Left lower pulmonary vein drains into LA through a narrow channel. Small Atrial Septal Defect (ASD) inhomogeneous opacity was evident in both lower lungs. CT diagnosis was a mixed type of supracardiac TAPVC with bilateral pulmonary inflammation. Operation was not done in this case.

\section{Case-3}

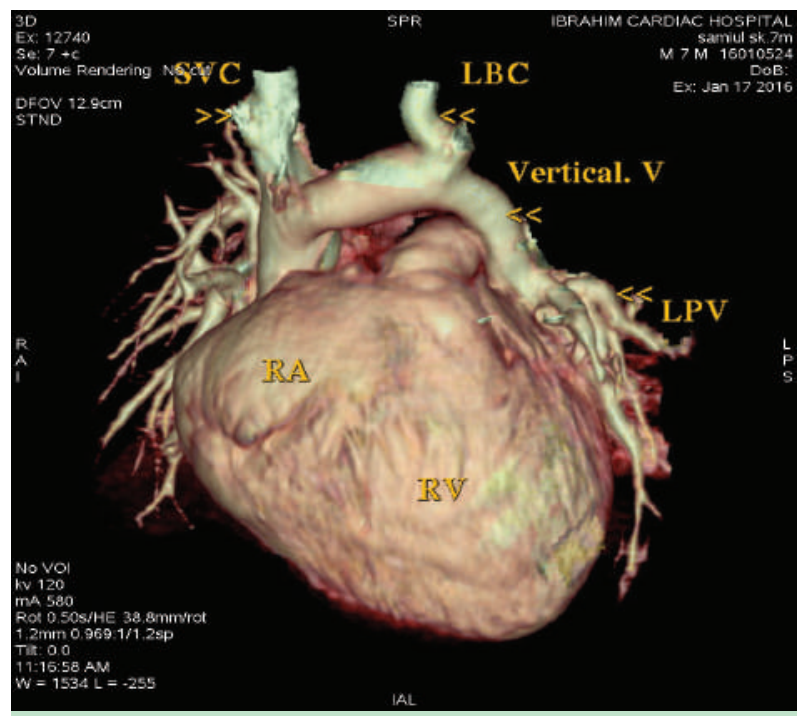

Figure : 3.1

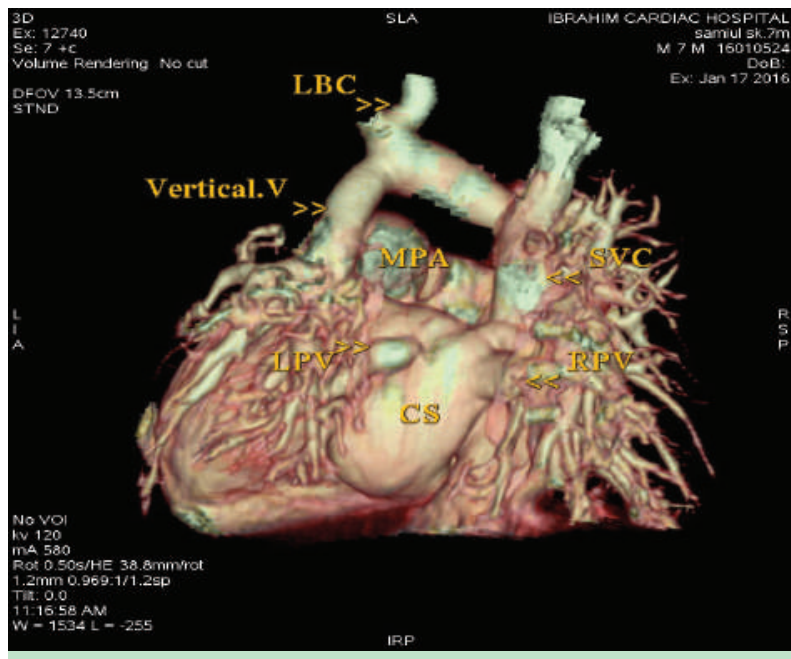

Figure : 3.2

Figure 3.1 \& 3.2: Anterior oblique \& posterior 3D volume-rendered (VRT) image shows two Right pulmonary veins form a confluence, which in turn, drains into the dilated coronary sinus, left upper pulmonary vein drains into SVC as vertical vein mixed type TAPVC.

\section{Discussion:}

Total anomalous pulmonary venous circulation (TAPVC) is an uncommon congenital heart defect. It consists of an abnormality of blood flow in which all 4 pulmonary veins drain into systemic veins or the right atrium with or without pulmonary venous obstruction. TAPVC is seen in nearly $1.5 \%$ of all patients with cardiovascular malformation and 6.8 per 1,000 live births. ${ }^{6}$ There are four variants of supracardiac TAPVC: blood drains to one of the innominate veins (brachiocephalic veins) or the superior vena cava (50\%); cardiac (20\%), where blood drains into coronary sinus or directly into right atrium; infradiaphragmatic (20\%), where blood drains into portal or hepatic veins; and a mixed $(10 \%)$ variant. ${ }^{7}$ Major advancements in the surgical and medical management of cases with pulmonary venous anomalies have allowed a large percentage of these cases to survive to adulthood. This required proper diagnosis and timed management to provide the child with a good chance of leading normal life with minimal (if any) disability. 
The clinical presentation is different if the pulmonary venous drainage is unobstructed (heart failure, mild cyanosis) or obstructed (respiratory failure, severe heart failure). Surgical management depends on the anatomic type. Obstructed TAPVC requires urgent surgical intervention, whereas unobstructed TAPVC can be dealt with electively. Although echocardiography is not a suitable modality for diagnosis of these patients with fair degree of accuracy, its importance cannot be ignored for initial assessment because of its mobility and availability. Echo has become the mainstay of diagnosis and follow-up for many congenital heart diseases, and is frequently used to determine chamber pressures, oxygen saturation, and pulmonary vascular resistance. However, it is usually limited by the acoustic window, spatial resolution and the subjective interpretation bias. The evaluation of the draining vein is not easy because the vein is located far from the anterior chest wall.8,9 However, it could be considered as an initial screening and diagnostic modality in patients with total anomalous pulmonary venous connection.

With the introduction of Multislice CT it has increasingly been used to diagnose complex cardiac anomalies. The latest Multislice CT has high spatial and temporal resolution and used in the diagnosis of many congenital anomalies, including those affecting the pulmonary veins. ${ }^{10}$ An accurate, 3D evaluation of the cardiac and related arterial anatomy is critical for the clinical management of adult and pediatric patients with complex congenital heart disease. 3D imaging has to be able to demonstrate the shapes of, and spatial relationships between the great arteries, proximal branch pulmonary arteries, and anomalous pulmonary venous or systemic connections. Main advantages of Multislice CT angiogram in congenital cardiac anomalies like TAPVC are the relative ease and accuracy with which the diagnosis can be made. 3D rendering and the multiplanar images give a clear picture to the surgeons of what they intend to find on the operating table. TAPVC is often associated with the other congenital anomalies and these are well-delineated by $3 D$ and maximum intensity projection (MIP) images.

\section{Conclusion:}

Multislice CT scan is an excellent modality for diagnosing complex congenital cardiac anomalies like TAPVC. The 3D and MIP images give a clear picture of TAPVC. Three-dimensional information about extra-cardiac morphological characteristics may determine subsequent surgical intervention, follow up the residuals of interventions, and assist with estimation of prognosis.

\section{Reference:}

1. Allen D. Total Anomalous Pulmonary Venous Connection Updated: May 02, 2015. Cited: 14/02/2017 8:55:47 AM. Available at: http://emedicine.medscape.com/article/899491-overview

2. Webb GD, Smallhorn JF, Therrien J \& Redington AN. Congenital heart disease. In: Bonow RO, Mann DL, Zipes DP, Libby $P$, eds. Braunwald's Heart Disease: A Textbook of Cardiovascular Medicine, $9^{\text {th }}$ ed. Philadelphia, Pa: Saunders Elsevier; 2011: chap 65.

3. Rhodes LA, Gustafson RA \& Phillips JP. The adult with congenital heart disease. $W V$ Med J 2006;102: L310-313.

4. Gilkeson RC, Zakha K \& Ciancibello L. Multidetector CT in evaluation of congenital heart disease in paediatric and adult patients. Am Heart J 2003;180(4):973-80.

5. Goo HW, Park IS, Ko JK, Kim YH, Seo DM \& Park JJ. Using multidetector-row $\mathrm{CT}$ in neonates with complex congenital heart disease to replace diagnostic cardiac catheterization for anatomical investigation: initial experiences in technical and clinical feasibility. Pediatr Radiol 2005;21:21-30.

6. corevillasenor A. Ferencz C, Boughman JA, Neil CA. Total anomalous pulmonary venous circulation; Familial \& environmental factors. Teratogy $1991 ; 44 ; 415-28$.

7. Alkhudhayri A, Shati AA, Broering DC, Azzi J \& Corno AF. Total Anomalous Pulmonary Venous Connection: Outcomes of Surgical Correction and Management in a New Saudi Center. Congenital Cardiology Today 2015:8-10.

8. Romero-Cardenas A, Vargas-Barron J, Rylaarsdam M, et al. Total anomalous pulmonary venous return: diagnosis by transesophageal echocardiography. Am Heart J 1991;121:1831-34.

9. Hsu YH, Chien CT, Hwang M, Chiu IS. Magnetic resonance imaging of total anomalous pulmonary venous drainage. Am Heart J 1991;125:1560-65.

10. Kim TH, Kim YM, Suh $\mathrm{CH}$, Cho DJ, Park Is, Kim WH, et al. Helical CT angiography and three dimensional reconstructions of total anomalous pulmonary venous connections in neonates and infants. AJR Am J Roentgenol 2000;175:1381-86. 